\title{
Cadherin expression in gastrointestinal tract endometriosis: possible role in deep tissue invasion and development of malignancy
}

\author{
Katy Van Patten, Vinita Parkash and Dhanpat Jain \\ Department of Pathology, Yale University School of Medicine, New Haven, CT, USA
}

\begin{abstract}
Cadherins are cell surface proteins crucial for cell adhesion and tissue integrity. The mechanism of deep tissue invasion in gastrointestinal endometriosis is unknown and may be related to the altered expression of these cell surface proteins. The goal of this study was to evaluate the expression of $\mathrm{N}$-cadherin, $\mathrm{E}$-cadherin, and $\beta$-catenin in peritoneal endometriotic implants, gastrointestinal endometriosis, and carcinoma arising in gastrointestinal endometriosis. Cases of peritoneal endometriosis, gastrointestinal endometriosis, and carcinoma arising in gastrointestinal endometriosis were identified from our pathology database. Immunohistochemistry was performed using antibodies against $\mathrm{N}$-cadherin, $\mathrm{E}$-cadherin, and $\beta$-catenin on representative tissue sections. Cases of normal proliferative and secretory endometrium and adenomyosis were included in the study for comparison. The intensity and extent of staining for each marker was scored semiquantitatively. Appropriate positive and negative controls were used. A total of 38 cases (peritoneal endometriosis $(n=14)$, gastrointestinal endometriosis ( $n=21$ : 11 colon, 8 appendix, 2 small bowel), and 3 cases of endometrioid carcinoma arising in colonic endometriosis $(n=3)$ ) were included in the study. Compared with normal proliferative endometrium, $\mathrm{N}$-cadherin expression was decreased in intensity and extent in secretory endometrium. Peritoneal and gastrointestinal endometriosis also showed markedly decreased expression of $\mathrm{N}$-cadherin compared with proliferative endometrium. All three cases of carcinoma arising in colonic endometriosis showed a total loss of $\mathrm{N}$-cadherin in the tumor, but preserved $\mathrm{E}$-cadherin and $\beta$-catenin expression. In these cases, areas of benign endometriotic glands near the tumor showed weak and focal $\mathrm{N}$-cadherin expression that was gradually lost. Moderate-to-strong membranous staining for $\beta$-catenin expression and variable intensity of E-cadherin expression was seen diffusely in normal endometrium and all study cases. These results strongly suggest that alterations of $\mathrm{N}$-cadherin expression in gastrointestinal endometriosis may have an important role in the mechanism that underlies deep tissue invasion, and possibly also in the development of malignancy. Modern Pathology (2010) 23, 38-44; doi:10.1038/modpathol.2009.127; published online 6 November 2009
\end{abstract}

Keywords: gastrointestinal endometriosis; cadherin; catenin; NCAD

Endometriosis is defined as the presence of ectopic endometrial glands and stroma outside the uterine cavity. It is a common disease that affects $\sim 10 \%$ of women, predominantly in their reproductive years. ${ }^{1}$ Gastrointestinal involvement may exist in up to $37 \%$ of these women. ${ }^{2}$ The clinical presentations of gastrointestinal endometriosis are diverse, mimicking appendicitis, ischemia, Crohn's disease, or even malignancy. The symptoms depend on the location and the extent of bowel involvement. Patients with

Correspondence: Dr D Jain, MD, Department of Pathology, Yale University School of Medicine, PO Box 208023, 20 York St. EP 2, New Haven, CT 06520-8023, USA.

E-mail: dhanpat.jain@yale.edu

Received 27 April 2009; revised 19 July 2009; accepted 30 July 2009; published online 6 November 2009 serosal lesions may be asymptomatic, whereas those with deeply infiltrative lesions may present with obstructive symptoms. The disease can also mimic malignancy clinically and radiologically. ${ }^{3}$ Infiltrative endometriosis can cause significant morbidity and the mechanism of infiltration remains unclear. ${ }^{2}$ Rarely, malignant transformation may occur, in up to $1 \%$ of women with gastrointestinal endometriosis. ${ }^{4}$

Cadherins are cell surface proteins with crucial roles in tissue development and integrity. Both E-cadherin and $\mathrm{N}$-cadherin are type 1 cadherins that bind to $\beta$-catenin to create a regulatory complex in the adherens junction. ${ }^{5}$ These cadherins mediate strong cell-cell and cell-matrix adhesion in many types of mature tissues. Normal endometrium shows intense E-cadherin and $\beta$-catenin expression throughout the menstrual cycle. ${ }^{6,7}$ However, the 
expression of $\mathrm{N}$-cadherin in normal endometrium is more variable between the proliferative and secretory menstrual phases. ${ }^{6,8}$ The reported expression of $\mathrm{N}$-cadherin in the endometriotic lesions has been inconsistent. One study of peritoneal endometriosis showed no significant difference in E-cadherin and $\mathrm{N}$-cadherin expression compared with normal endometrium, whereas others have shown varying $\mathrm{N}$-cadherin expression between endometriosis and normal endometrium. ${ }^{6,7}$

Cadherin and $\beta$-catenin expression have not been previously evaluated in gastrointestinal endometriosis that deeply infiltrates the wall. The mechanism of this infiltration is unknown and may be related to abnormal expression of these adhesion molecules. The goal of this study was to evaluate the expression of $\mathrm{N}$-cadherin, E-cadherin, and $\beta$-catenin in cases of gastrointestinal and peritoneal endometriosis, and compare the results with the expression in normal endometrium. We also wanted to compare the results with the expression of these adhesion molecules in malignant transformation of gastrointestinal endometriosis.

\section{Materials and methods}

The pathology database at our institution was searched for patients with peritoneal endometriotic implants, gastrointestinal endometriosis, and gastrointestinal endometriosis with malignant transformation from 1999 to 2008. Thirty-eight patients with available tissue blocks were included in the study. Slides were reviewed to confirm the diagnosis. The depth of involvement by gastrointestinal endometriosis (mucosa, submucosa, muscularis propria, or serosa) was evaluated in each case. Pertinent clinical and demographic information was recorded for all study cases. Endometrial samples of normal proliferative $(n=10)$, secretory endometrium $(n=16)$, and adenomyosis $(n=13)$ from 39 age-matched women who underwent hysterectomy for benign conditions were also included for comparison.

Immunohistochemical analysis was performed on $4-\mu \mathrm{m}$ thick, formalin-fixed, paraffin-embedded representative tissue sections from each case using an indirect immunoperoxidase technique and using antibodies against E-cadherin, N-cadherin, and $\beta$-catenin. (Table 1) Appropriate positive and negative controls were used with each antibody.
For each antibody, the percentage of the glands with partial and complete membranous staining were evaluated and scored as $0=$ none, $1=1-33 \%$, $2=34-66 \%$, and $3=67-100 \%$. The intensity of the membranous staining was scored as $0=$ none, $1+$ $=$ faint staining in part of the cell membrane, $2+$ $=$ weak-to-moderate staining of the entire cell membrane, and $3+=$ strong staining of entire cell membrane. The scores for both parameters were combined for each sample to obtain a final score for each marker.

\section{Results}

The clinicopathological and demographic data for these patients are shown in Table 2. Twentyone cases involved benign gastrointestinal endometriosis $($ colon $=11$, appendix $=8$, and small bowel $=2$ ). The patient age range was 32-84 years (mean 45 years). Most of the patients $(n=19,90 \%)$ were in the premenopausal or perimenopausal stage. The endometriosis infiltrated the mucosa in 1 case $(5 \%)$, the submucosa in $7(33 \%)$, the muscularis propria in $10(48 \%)$, and only serosa in

Table 2 Characteristics of patients with benign gastrointestinal endometriosis (21 patients)

\begin{tabular}{lr}
\hline Characteristic & Cases \\
\hline Age (years) & \\
$30-34$ & 4 \\
$35-44$ & 8 \\
$45-54$ & 7 \\
$55+$ & 2 \\
Clinical symptoms & \\
None & 1 \\
Abdominal pain & 7 \\
Symptoms related to coinciding disease & 11 \\
Endometriosis history & 3 \\
& \\
Site of involvement & 2 \\
Small bowel & 10 \\
Large bowel & 9 \\
Appendix & \\
Depth of involvement & 3 \\
Serosa & 10 \\
Muscularis propria & 7 \\
Submucosa & 1 \\
Mucosa &
\end{tabular}

Table 1 Antibodies used

\begin{tabular}{llll}
\hline Antibody & N-cadherin & E-cadherin & $\beta$-catenin \\
\hline Dilution & $1: 100$ & $1: 40$ & $1: 25$ \\
Clone & $6 G 11$ & NCH-38 & $\beta$-catenin-1 \\
Manufacturer & Dako & Dako & Dako \\
Location & Carpinteria, CA, USA & Carpinteria, CA, USA & Carpinteria, CA, USA \\
Antigen retrieval & Dako high pH & Dako low pH & Dako low pH \\
Retrieval time & 20 min & 30 min & 20 min \\
\hline
\end{tabular}


Table 3 N-cadherin combined score (intensity score+percentage score)

\begin{tabular}{|c|c|c|c|c|c|c|}
\hline$N$-cadherin total score & 0 & $1-2$ & $3-4$ & $5-6$ & Mean $\pm s . d$. & $\mathrm{P}$-valu \\
\hline Proliferative endometrium & 0 & 2 & 3 & 5 & $4.2 \pm 1.3$ & \\
\hline $\begin{array}{l}\text { Gastrointestinal } \\
\text { endometriosis }\end{array}$ & 3 & 6 & 8 & 4 & & \\
\hline Peritoneal endometriosis & 3 & 8 & 1 & 1 & & $<0.0$ \\
\hline Aden & 2 & 3 & 5 & & & \\
\hline $\begin{array}{l}\text { Carcinoma in } \\
\text { endometriosis }\end{array}$ & 3 & 0 & 0 & 0 & $0 \pm 0$ & $<0.0$ \\
\hline Secretory endometrium & 4 & 9 & 2 & 0 & $1.5 \pm 1.1$ & $<0.0$ \\
\hline
\end{tabular}

$3(14 \%)$ cases. Fourteen cases involved peritoneal endometriotic implants only. The patient age range for these cases was 24-51 years (mean 39 years) and all of the patients were in the premenopausal or perimenopausal stage. The three patients with malignant transformation in gastrointestinal endometriosis were 41,51 , and 61 years of age.

The N-cadherin total scores for the normal endometrium are shown in Table 3 and Figure 1. Normal proliferative endometrium had $2+$ to $3+$ staining of $\mathrm{N}$-cadherin in more than $30 \%$ of the glands in 8 of 10 cases. Secretory endometrium had a decreased extent (5-10\% of glands) and intensity of staining $(1+$ to $2+)$ in all the 16 cases was studied. Cases of adenomyosis had more variable staining with decreased intensity of $\mathrm{N}$-cadherin expression in 8 of 13 cases with 0 to $1+$ staining.

The results of immunohistochemical staining of the benign gastrointestinal endometriosis cases are shown in Table 3 and Figure 2 . Strong $(2+$ to $3+$ ) membranous $\beta$-catenin staining was present in all glands in almost all benign gastrointestinal endometriosis cases $(n=20,95 \%)$ and controls, including normal endometrium. One case showed weak $(1+)$ but diffuse staining in all endometriotic glands. None of the cases and controls showed nuclear staining for $\beta$-catenin. E-cadherin was present in all cases of gastrointestinal and peritoneal endometriosis and controls, including normal endometrium, and the immunoreactivity was strong in most cases of gastrointestinal endometriosis $(76 \%)$ and controls. There was little to no N-cadherin expression in $13(62 \%)$ of the gastrointestinal and 13 $(87 \%)$ of peritoneal endometriosis cases. The three cases of gastrointestinal endometriosis with malignant transformation all showed similar findings. There was $1+$ to $2+$ focal staining in some of the benign glands adjacent to the tumor and no staining in the tumor itself. (Figure 3) Many of the endometriotic glands showed no staining. Areas of transition between benign endometriotic glands and carcinoma showed gradual loss of N-cadherin.

\section{Discussion}

The most widely accepted theory regarding the pathogenesis of endometriosis involves the reflux of menstrual tissue through the ostia of the fallopian tubes with subsequent peritoneal implantation. ${ }^{9}$ This retrograde menstrual flow has been clinically identified in women who had abdominal surgery during menstruation. ${ }^{10,11}$ Interestingly, endometriosis outsidethe abdominal cavity, such as in the nasal cavity and brain, has also been described and is difficult to explain by this simplistic mechanism. ${ }^{12,13}$ Another explanation includes the 'metaplasia theory', which proposes that an as-yet undetermined stimulus induces metaplasia of the visceral and abdominal peritoneum resulting in endometriosis. ${ }^{14,15}$ Other theories include development of embryonic rests into endometriotic lesions and the metastasis of endometriotic tissue through the lymphovascular system. ${ }^{16,17}$

Although endometriosis is a benign lesion, it often displays characteristics that are associated with malignancy. It can invade into the abdominal tissues and the viscera and has a 'metastatic pattern' of spread. In addition, studies have shown that endometriotic lesions are monoclonal and analogous to neoplastic proliferations. ${ }^{18}$ A recent study using the HUMARA assay for the X-chromosome inactivation pattern showed that all endometriotic foci $(n=40)$ were monoclonal, and each focus represented a separate clone. ${ }^{19}$ The investigators suggested that after fragments of endometrium are shed, single cells might implant and develop individual monoclonal endometriotic foci. Results of the evaluation of loss of heterozygosity (LOH) in endometriosis have been less definitive. A metaanalysis of LOH studies of endometriosis showed markedly variable results, ranging from none of the cases to $56 \%$ of cases. ${ }^{18} \mathrm{~A}$ more recent study of ovarian endometriosis found no $\mathrm{LOH}^{20}$ Thus far, despite some similarities to malignancy, molecular evidence of the neoplastic nature of endometriosis is inconclusive and the mechanism of invasive endometriosis remains unclear.

Among the cell adhesion molecules, E-cadherin and $\mathrm{N}$-cadherin have critical roles in maintaining normal tissue structure and function. E-cadherin has a role in cell adhesion in the epithelial cells and $\mathrm{N}$-cadherin is, in general, a mesenchymal cadherin that promotes cell motility. N-cadherin, in addition, is also a regulator of apoptosis and some investigators have shown that dominant-negative N-cadherin mutants have an increase in apoptosis and that $\mathrm{N}$-cadherin binding prevents apoptosis. ${ }^{21,22}$ Our study showed strong diffuse $\mathrm{N}$-cadherin staining in the endometrial glands in the proliferative phase and minimal staining in the secretory phase of normal endometrium, findings akin to other recent studies. ${ }^{6,8}$ This suggests that $\mathrm{N}$-cadherin helps maintain the integrity of the endometrium during the proliferative phase and that its decreased expression during the secretory phase may have a role in altering cell-cell adhesion so as to allow for the implantation of the embryo. Later, in the absence of implantation, the persistent loss of N-cadherin 

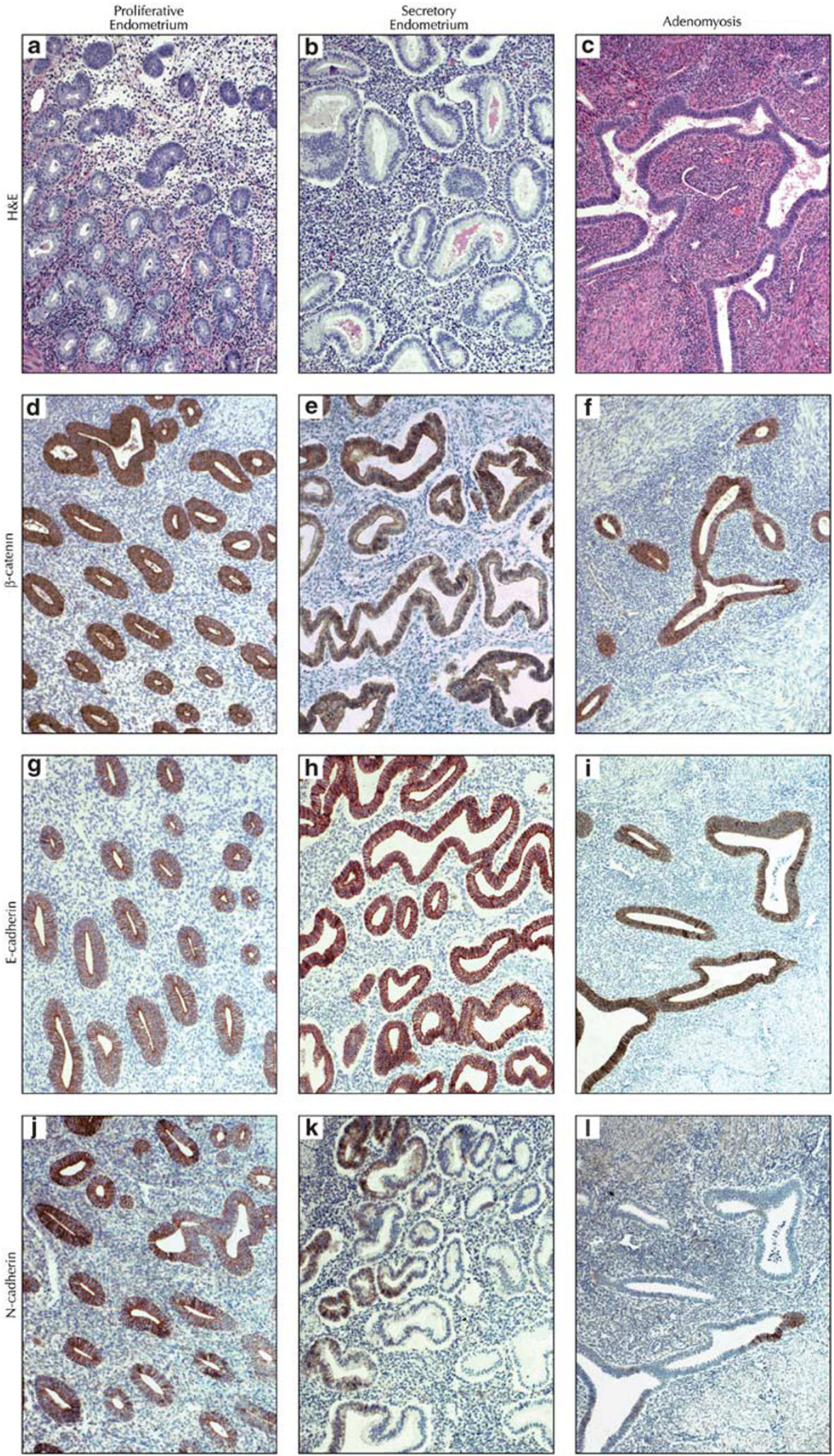

Figure 1 Hematoxylin and eosin staining of (a) proliferative endometrium, (b) secretory endometrium, and (c) adenomyosis. Diffuse staining of $\beta$-catenin in (d) proliferative endometrium, (e) secretory endometrium, (f) and adenomyosis. Diffuse staining of E-cadherin in (g) proliferative endometrium, (h) secretory endometrium, and (i) adenomyosis. N-cadherin staining with (j) diffuse staining in proliferative endometrium, (k) staining limited to basal glands in secretory endometrium, and (l) focal staining of a single gland in adenomyosis. 

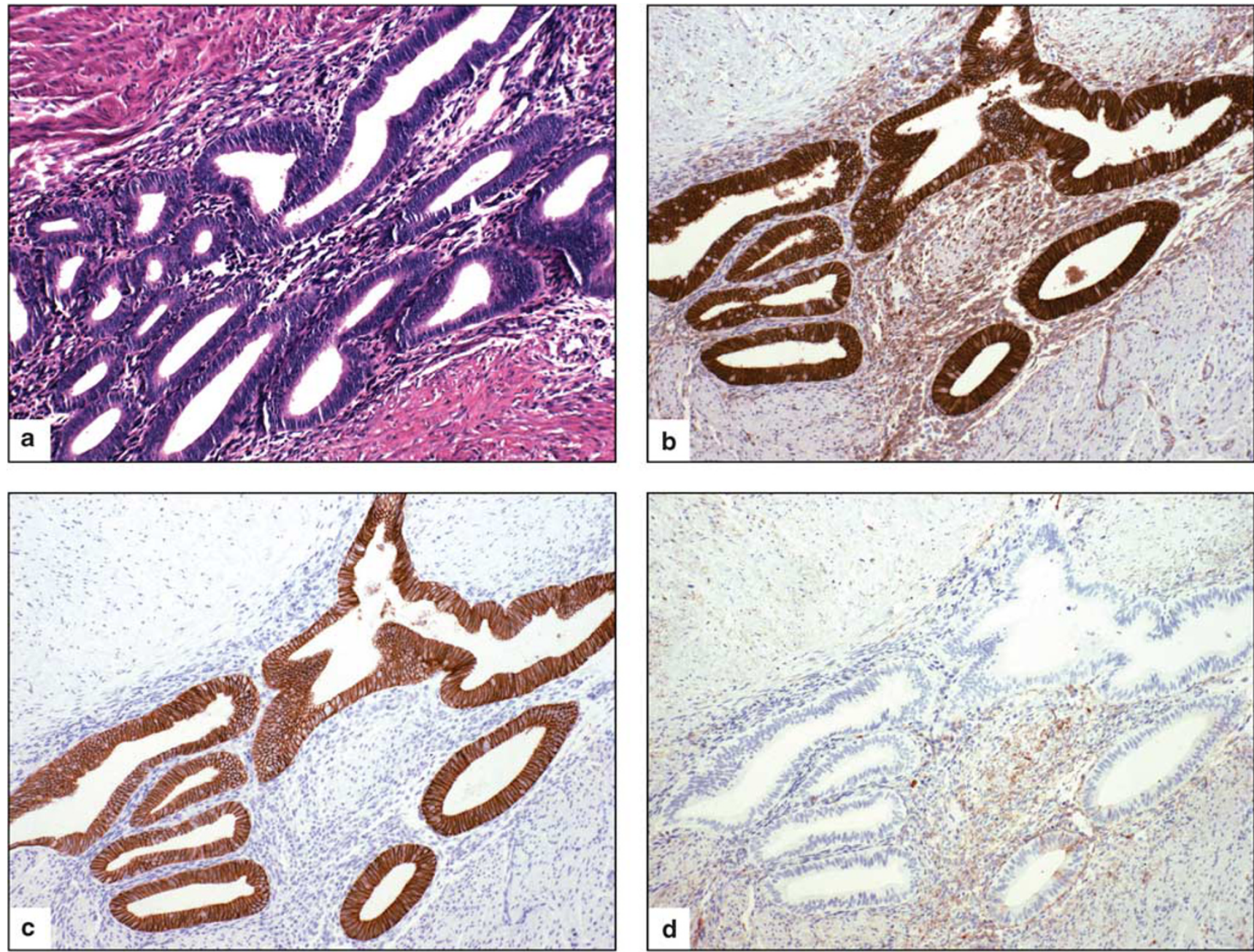

Figure 2 Gastrointestinal endometriosis staining with (a) hematoxylin and eosin, (b) $\beta$-catenin and (c) E-cadherin showing diffuse staining, and (d) N-cadherin showing no staining of endometriotic glands.

may allow for increasing apoptosis and eventually shedding of the endometrium (menstruation).

It is the alterations in the expression of E-cadherin and $\mathrm{N}$-cadherin that further reveal the additional functions of these proteins. E-cadherin has been shown to have a role as a tumor suppressor and a regulator of cell polarity. ${ }^{23}$ The loss of E-cadherinmediated cell adhesion is considered a feature of epithelial-to-mesenchymal transformation. ${ }^{24,25}$ Alterations in N-cadherin expression in epithelial cells have also been shown to change the metastatic ability of some types of tumor cells. The switch from E-cadherin to $\mathrm{N}$-cadherin expression has been shown to promote motility and invasion in several types of malignancies, including breast and prostate carcinoma, and melanoma. ${ }^{26-28}$

However, one crucial feature of this finding is that this change in cadherin expression and the subsequent alteration in cell motility have been described only in the context of tissues that do not normally express N-cadherin. In normal tissues that do express N-cadherin, such as the endometrium, it is possible that the change in ratio between the various cadherins may confer a more infiltrative behavior. In settings in which expression is not lost from benign to malignant transformation, further investigation is warranted with regard to other adhesion molecules. In one study of 225 endometrial carcinomas, the majority of the endometrioid variants did retain E-cadherin expression. ${ }^{29}$ Our cases of carcinoma arising in endometriosis also retained the E-cadherin expression but showed complete loss of N-cadherin. Although the full significance of this observation is still unclear, further investigation is warranted. This alteration in $\mathrm{N}$-cadherin, and not E-cadherin, may have a role in the infiltrative behavior of these lesions.

The cadherin expression in peritoneal endometriotic lesions in our study differs from previous studies. One recent study showed no significant difference in E-cadherin or $\mathrm{N}$-cadherin expression in peritoneal endometriosis when compared with the normal endometrium. ${ }^{6}$ Our results showed that compared with normal proliferative endometrium, $\mathrm{N}$-cadherin expression in peritoneal endometriosis was markedly decreased, both in the intensity and 

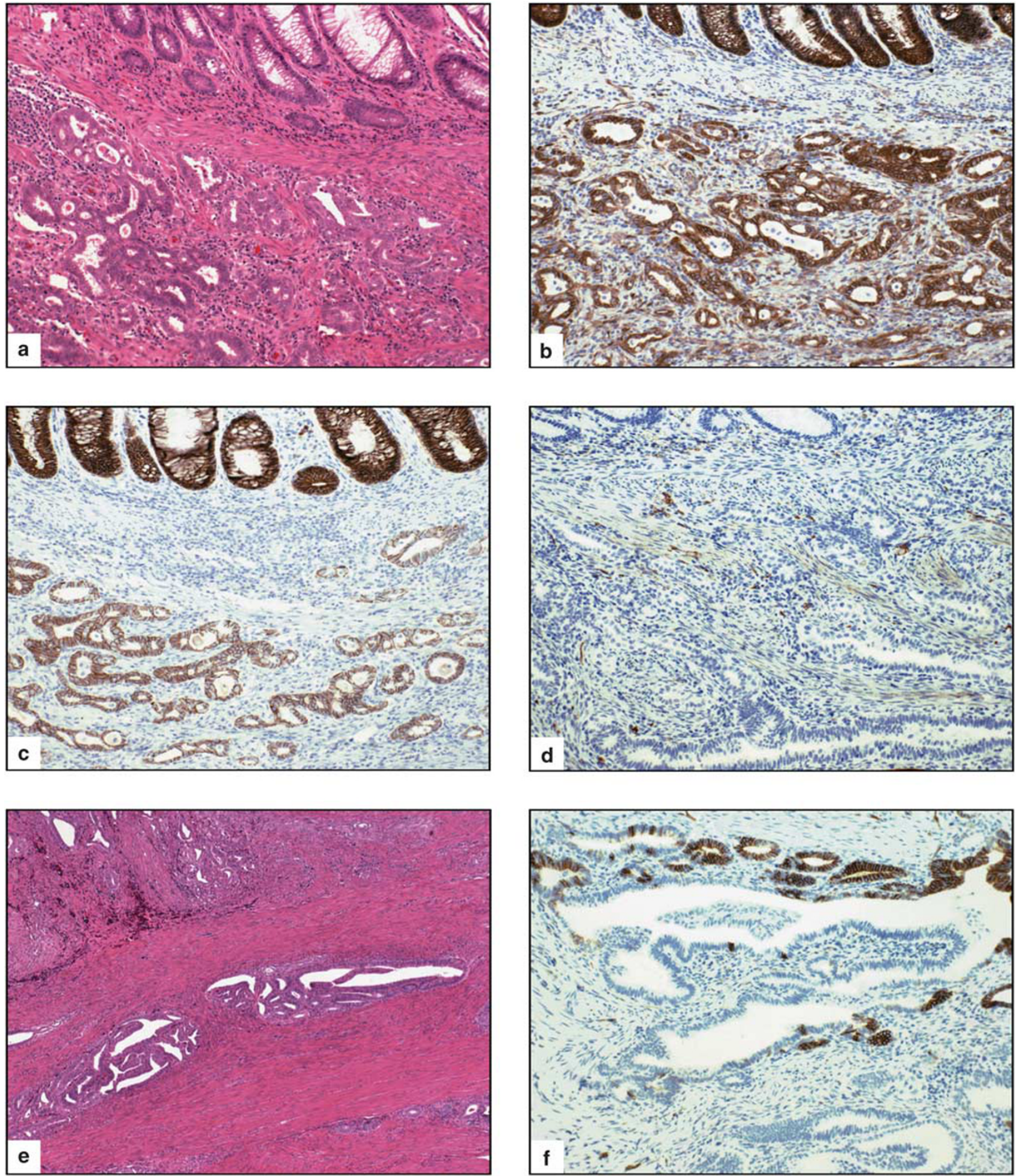

Figure 3 Case of carcinoma arising in gastrointestinal endometriosis staining with (a) hematoxylin and eosin, (b) $\beta$-catenin and (c) Ecadherin showing diffuse staining, and (d) N-cadherin showing no staining in tumor. The adjacent benign endometriotic glands are shown staining with (e) hematoxylin and eosin and (f) N-cadherin with only focal staining.

extent of staining. The more invasive gastrointestinal lesions, particularly those that invaded into the muscularis propria and beyond, also had markedly decreased expression compared with proliferative endometrium. Interestingly, even the endometriotic glands infiltrating within the myometrium (adenomyosis) also showed reduced expression of $\mathrm{N}$-cadherin compared with normal.

Another factor that may also contribute to decreased expression of $\mathrm{N}$-cadherin in various 
lesions may be cyclical changes in $\mathrm{N}$-cadherin expression with the menstrual cycle. The information regarding the phase of the menstrual cycle was not available in any of our cases; however, our findings with regard to the deeply invasive gastrointestinal endometriosis were consistent irrespective of age or menopausal status. Thus the changes in the expression of $\mathrm{N}$-cadherin seen in our study in endometriotic lesions are unlikely to be solely due to changes with the menstrual cycle.

These results strongly suggest that decreased expression of $\mathrm{N}$-cadherin may have an important role in the shedding of endometrial glandular cells during the menstrual cycle, peritoneal implantation of endometrial glands, infiltrative growth, and the development of malignancy. The underlying molecular mechanisms leading to the more infiltrative gastrointestinal lesions may be crucial to our understanding of endometriosis and the subsequent development of malignancy. Further studies evaluating these mechanisms would also be important in our understanding of malignant transformation of endometriosis.

\section{Disclosure/conflict of interest}

The authors declare no conflict of interest.

\section{References}

1 Scotti S, Regidor PA, Schindler AE, et al. Reduced proliferation and cell adhesion in endometriosis. Mol Hum Reprod 2000;6:610-617.

2 Yantiss RK, Clement PB, Young RH. Endometriosis of the intestinal tract: a study of 44 cases of a disease that may cause diverse challenges in clinical and pathologic evaluation. Am J Surg Pathol 2001;25:445-454.

3 Pickhardt PJ, Kim DH, Menias CO, et al. Evaluation of submucosal lesions of the large intestine: part 2 . Nonneoplastic causes. Radiographics 2007;27: 1693-1703.

4 Lauslahti K. Malignant external endometriosis. Obstet Gynecol 1972;2:283-289.

5 Goodwin M, Yap AS. Classical cadherin adhesion molecules: coordinating cell adhesion, signaling and the cytoskeleton. J Mol Histol 2004;35:839-844.

6 Poncelet C, Leblanc M, Walker-Combrouze F, et al. Expression of cadherins and CD44 isoforms in human endometrium and peritoneal endometriosis. Acta Obstet Gynecol Scand 2002;81:195-203.

7 van der Linden PJ, de Goeij AF, Dunselman GA, et al. P-cadherin expression in human endometrium and endometriosis. Gynecol Obstet Invest 1994;38: 183-185.

8 Tsuchiya B, Sato Y, Kameya T, et al. Differential expression of $\mathrm{N}$-cadherin and E-cadherin in normal human tissues. Arch Histol Cytol 2006;69:135-145.

9 Sampson JA. Peritoneal endometriosis due to menstrual dissemination of endometrial tissue into the peritoneal cavity. Am J Obstet Gynecol 1927;14:422-469.

10 Liu DT, Hitchcock A. Endometriosis: its association with retrograde menstruation, dysmenorrhoea and tubal pathology. Br J Obstet Gynaecol 1986;93:859-862.
11 Watkins RE. Uterine retrodisplacements, retrograde menstruation and endometriosis. West J Surg Obstet 1938;46:480-494.

12 Laghzaoui O, Laghzaoui M. [Nasal endometriosis: apropos of 1 case]. J Gynecol Obstet Biol Reprod (Paris) 2001;30:786-788.

13 Thibodeau LL, Prioleau GR, Manuelidis EE, et al. Cerebral endometriosis. Case report. J Neurosurg 1987; 66:609-610.

14 Gardner G, Greene RR, Ranney B. The histogenesis of endometriosis: a review of facts and fancies. Obstet Gynecol 1953;1:615.

15 Gruenwald P. Origin of endometriosis from the mesenchyme of the coelomic walls. Am J Obstet Gynecol 1942;44:470.

16 Russell WW. Aberrent portions of the mullerian duct found in an ovary: ovarian cysts of mullerian origin. Bull John Hopkins Hospital 1899;10:8-10.

17 Sampson JA. Heterotopic or misplaced endometrial tissue. Am J Obstet Gynecol 1925;10:649-664.

18 Vigano P, Somigliana E, Chiodo I, et al. Molecular mechanisms and biological plausibility underlying the malignant transformation of endometriosis: a critical analysis. Hum Reprod Update 2006;12:77-89.

$19 \mathrm{Wu}$ Y, Basir Z, Kajdacsy-Balla A, et al. Resolution of clonal origins for endometriotic lesions using laser capture microdissection and the human androgen receptor (HUMARA) assay. Fertil Steril 2003;79(Suppl 1):710-717.

20 Prowse AH, Fakis G, Manek S, et al. Allelic loss studies do not provide evidence for the 'endometriosis-as-tumor' theory. Fertil Steril 2005;83(Suppl 1): 1134-1143.

21 Hermiston ML, Wong MH, Gordon JI. Forced expression of E-cadherin in the mouse intestinal epithelium slows cell migration and provides evidence for nonautonomous regulation of cell fate in a self-renewing system. Genes Dev 1996;10:985-996.

22 Trolice MP, Pappalardo A, Peluso JJ. Basic fibroblast growth factor and $\mathrm{N}$-cadherin maintain rat granulosa cell and ovarian surface epithelial cell viability by stimulating the tyrosine phosphorylation of the fibroblast growth factor receptors. Endocrinology 1997;138:107-113.

23 Wheelock MJ, Jensen PJ. Regulation of keratinocyte intercellular junction organization and epidermal morphogenesis by E-cadherin. J Cell Biol 1992;117: 415-425.

24 Delva E, Kowalczyk AP. Regulation of cadherin trafficking. Traffic 2009;10:259-267.

25 Stemmler MP. Cadherins in development and cancer. Mol Biosyst 2008;4:835-850.

26 Hazan RB, Phillips GR, Qiao RF, et al. Exogenous expression of $\mathrm{N}$-cadherin in breast cancer cells induces cell migration, invasion, and metastasis. J Cell Biol 2000;148:779-790.

27 Tomita K, van Bokhoven A, van Leenders GJ, et al. Cadherin switching in human prostate cancer progression. Cancer Res 2000;60:3650-3654.

28 Qi J, Chen N, Wang J, et al. Transendothelial migration of melanoma cells involves $\mathrm{N}$-cadherin-mediated adhesion and activation of the beta-catenin signaling pathway. Mol Biol Cell 2005;16:4386-4397.

29 Scholten AN, Aliredjo R, Creutzberg CL, et al. Combined E-cadherin, alpha-catenin, and beta-catenin expression is a favorable prognostic factor in endometrial carcinoma. Int J Gynecol Cancer 2006;16: 1379-1385. 